\title{
Second-Order Cone Programming Based Robust Blind Multiuser Detector for CDMA Systems
}

\author{
Zhongqiang Luo ${ }^{*}$ and Chengjie $\mathrm{Li}$ \\ National Key Lab. of Science and Technology on Communications, \\ University of Electronic Science and Technology of China, \\ Chengdu 611731, China \\ E-mail:"luozhongqiang@126.com
}

\begin{abstract}
In this paper, we propose a robust blind multiuser detector for code-division multiple access (CDMA) systems against signature waveform mismatch (SWM) derived from the influences of time asynchronization or channel distortion. This blind detection method with SWM problem is formulated as blind source separation (BSS) model subject to the second-order cone (SOC) constraint. The resulting blind separation based on SOC programming problem is solved by approximate negentropy maximization using quasiNewton iterative methods. Theoretical analysis and simulation results show that the performance of the proposed blind detector is superior to those of the existing methods.
\end{abstract}

Keywords: Code division multiple access (CDMA); Second-order cone programming; Blind source separation (BSS); Blind multiuser detector; Independent Component Analysis (ICA)

\section{Introduction}

In the past few years, blind source separation (BSS) technique has received considerable interests for its potential application to wireless communication systems [130]. Especially, the research work of CDMA system with BSS technology is of great importance for anti-jamming in military communications (MILCOM) and satellite communications (SATCOM). The signal separation problem is decorated "blind" to reflect the lack of information concerning the source signals and mixing matrix (i.e. channel condition). The only prior information utilized is the often soundly justified assumption of statistical independence between the source signals. Thus, BSS is also known as independent component analysis (ICA) [1-3]. ICA is a statistical technique where the goal is to represent a set of random variables as a linear transformation of statistically independent component variables. By virtue of the ICA technique, the observed mixed signal can be decomposed into as a representation of meaningful sources, and the sources of interest can be extracted and recovered.

There are some motivating reasons to use the means of ICA in the reception of a CDMA system [8-9]. First of all, ICA provides a near-far resistant receiver, being able to resist strong interferences. Since ICA only requires the source signals to be statistically independent, but their strengths are allowed to differ. Secondly, ICA provides robustness against erroneous parameter estimation. Since ICA does not need that precise knowledge of the system's parameters due to blind properties. Thirdly, ICA technique can combine with the existing receiver structure to establish hybrid receiver structures, which can be intelligently activated to improve performance. At last, the spectrum effectiveness of CDMA system can be enhanced due to eliminating (long) pilot sequence. That is to say, the crucial factor of employing blind detector is to avoid the requirements of a training sequence and the reduced loss of channel throughput. Therefore, blind detector is 
attractive for the high spectrum effectiveness need of the future wireless communications [31].

Blind multiuser detection (BMUD) is known as blind con-channel interference suppression or blind interference cancellation [15, 27]. It has important application to the channel problem suffered by time-variant multipath fading and multiple-access interference (MAI). As far as we are concerned, we can divide the existing BMUD method into three types of blind multiuser detectors for CDMA system. The first type of blind detectors is based on accurate knowledge of the channel impulse response or the signature waveform of the user of interest at the receiver [4-6]. However, in the practical circumstances, a perfect estimation of the information of channel parameters cannot be conveyed to the receiver, leading to the degraded performance of the system accordingly. For example, the constrained minimum output energy (CMOE) based linear minimum mean square error (LMMSE) blind detector is proposed by Honig et al. in [6]. However, due to the presence of channel and data correlation matrix estimation errors, this blind multiuser detector has descent performance. The second type of blind multiuser detectors is the subspace-based blind detectors [7-8]. Those blind receivers are always based on second-order statistic (SOS) using eigenvalue decomposition (EVD) on the covariance matrix of the received signal. However, in this type of blind detectors, the orthogonality between the signal and noise subspaces is suffered serious damage due to correlated noise subspace [8-9].

The third type of blind multiuser detectors is the ICA based BSS detection, which is also referred to as ICA-based receivers [10-26]. The higher-order statistic (HOS) based ICA algorithms are used to deal with BSS of unknown sources from a mixed-up received signal. The ICA-based receivers are based on the assumption of the independence and non-Gaussianity of the sources. In many ICA-based receivers, the approximate negentropy maximizing based fast independent component analysis (FastICA) [1-2] and fourth-order cumulant based joint approximate diagonalization of eigen-matrices (JADE) [2] are always used to carry out blind separation work. For ICA-based blind detectors, two major problems are investigated by many researchers, i.e. external interference cancellation problem and internal interference cancellation problem [10-26]. In the external interference cancellation problem, the CDMA signal and external interference signal are mutual independence, which is modeled as ICA model based on two-sensor array [10-14]. Then ICA algorithm is executed blind separation to suppress external interference, following that the separated CDMA signal is implemented conventional detection for the desired user signal. For example, the work is in [10-14]. In view of the internal interference cancellation problem, the structure of the CDMA channel where all the users transmit their data poses a similarity to the BSS problem and prompts the use of ICA in this scenario [15-26]. Then the blind separation work can be carried out so that each user signal can be separated from the mixed signals. For example, the related works are shown in literature [15-26].

However, the ICA technique has inherent ambiguity problem. The ambiguity feature of any ICA method is that it is able to estimate a set of independent source signals, but the order of those will be unpredictable. Therefore, with regard to ICA-based receivers, it is not meaningful to apply ICA (FastICA or JADE) on its own to implement separation work directly. In order to overcome this problem, the literature $[16,17]$ proposes that the ICA part is incorporated as an add on to the existing MMSE or rake receiver, i.e. MMSEICA and RAKE-ICA detectors. The two types of detectors are developed by modifying the classical FastICA algorithm based on the quasi-Newton iteration as well as the proper starting point initializing iterations. In literature [18] presents an algorithm to remove the indeterminacy in ICA solution by imposing a norm constraint based on the knowledge of the signature code of the desired user. The previous related ICA-based detectors first implement the centering and whitening steps and then select the appropriate starting point initializing iterations for obtaining the correct weight/filter vector. However, the above- 
mentioned ICA-based detectors are sensitive to the colored noise, which will lead to falling system's performance. The reason is that the whitening based eigenvector projection is unable to decorrelate the mixed-up signals. Likewise, the SOS-based technique will fail under colored noise environment since some of correlated noise subspace diverges into the signal eigenvectors causing serious destruction to this orthogonality especially at low SNR [27-29].

In the literature [26], the authors propose a robust blind multiuser detector for CDMA systems based on second-order cone (SOC) programming. In [26], the worst-case performance optimization is utilized to overcome the SWM problem. Furthermore, the SOC programming is incorporated to as an add on to JADE blind separation algorithm, i.e. SOC-JADE. In addition to the statistical properties of fourth-order cumulant, SOCJADE has robust performance to SWM and colored noise. However, this proposed algorithm has higher computation complexity. Especially, when the number of users in CDMA system is large, the separation work is too consuming to adapt the real-time requirement in communications. Therefore, motivated by the channel environment encountered in many CDMA applications, the design of new blind multiuser detectors is attractive and appealing to satisfy further anti-interference requirements.

In this paper, a new blind multiuser detector will be developed, which is based on approximate negentropy maximization subject to SOC constraint [26-28]. The proposed algorithm can be considered as a modified FastICA algorithm which is referred to as SOC-FastICA in this paper. The SOC-FastICA based blind detector is not only capable of dealing with the weight order ambiguity present in the classical ICA algorithm, converging on the correct solution subject to colored noise, but also has lower computation complexity. Theoretical analysis and computer simulations indicate that the performance of the proposed blind multiuser detectors is superior to those in existing literatures.

The remaining of this paper is organized as follows. In Section 2, the ICA technique is reviewed. Section 3 describes the signal model for the CDMA system. Section 4 illustrates the principle of blind detectors based on ICA and the proposed algorithm for blind multiuser detector respectively. Section 5 analyzes the computation complexities of proposed algorithm, and is compared with other existing blind detectors. Simulation results and discussions are shown in Section 6. Section 7 concludes this paper.

\section{Independent Component Analysis}

Independent component analysis (ICA) is a statistical technique which involves the task of computing the mixing projection of a set of components as a linear transformation of statistically independent component variables. The main application of ICA is blind separation problem, which has become an appealing field of research in statistical signal processing related communications [1, 24]. The increasing interest in ICA is mainly due to emerging new practical application areas, where the assumption of independence is both powerful and realistic, which making it possible to find meaningful source signals or independent component from the data to be analyzed in a completely blind manner [1618]. In the conventional ICA method, the data model is described as follows.

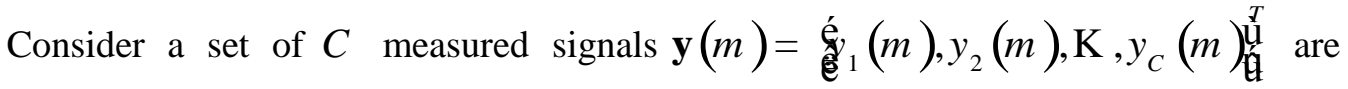
instantaneous linear combinations of a set of $K$ mutually independent unknown source

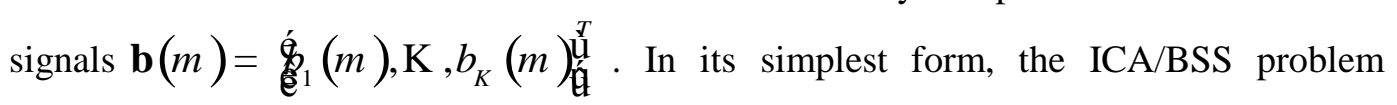
accepts the following matrix model.

$$
\mathbf{y}(m)=\mathbf{G b}(m)+\mathbf{n}(m)
$$

where $\mathbf{G}$ is an unknown full rank mixing matrix, $\mathbf{n}(\mathrm{m})$ is a realization of a noise process. 
If the mixing matrix $\mathbf{G}$ is non-singular, $\mathbf{y}(m)$ is a stationary ergodic random sequence, and no more than one Gaussian distributed sources is present in the mixture, forcing the statistical independence of the outputs yields the sources as follows

$$
\mathbf{z}(m)=\mathbf{W y}(m)
$$

where $\mathbf{W}$ is referred to as separation matrix. Since the scale and the order of the components of $\mathbf{b}(\mathrm{m})$ do not affect their statistical independence, satisfactory separation is characterized by a global matrix $\mathbf{C}=\mathbf{W A}$ with a non-mixing structure, that is, with a single non-null element per row and per column. This is an inherent feature of any ICA method is that the original scaling and arrangement cannot be estimated from the mere independence assumption. Although it is unimportant in blind separation problem, it should not to be ignored in communication systems. A schematic description of the ICA mathematical model is shown in Figure1.

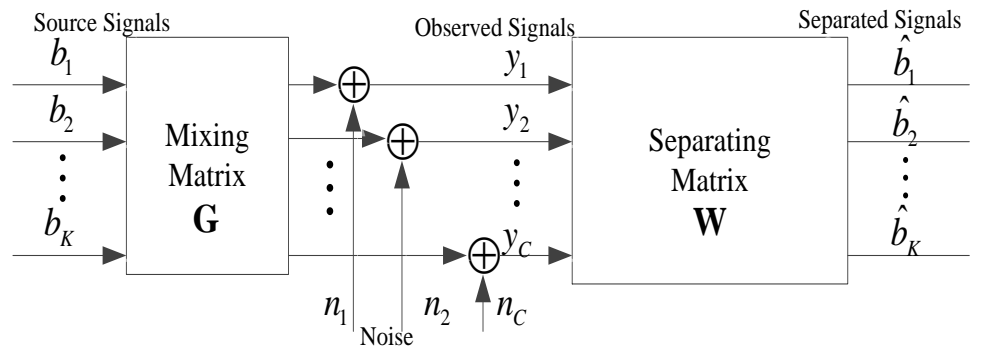

Figure 1. Schematic Description of the ICA Model

\section{CDMA System Model}

A CDMA channel is characterized by the fact that there is no separation between the users in the frequency domain or in the time domain. In CDMA, each user uses its own code sequence (or signature sequence) to spread its narrowband information signal before transmission. The signature sequence is user specific, thus identifies each user in the system. The signal received at the receiver in continuous time domain can be represented as

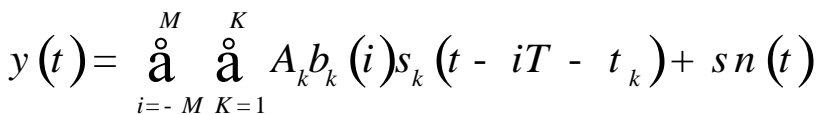

where $y(t)$ is the received signal; $A_{k}$ is the $k$ th user's channel gain; $b_{k}(i) \hat{I}$ é $1,+1$ ù is the $i$ th data symbol transmitted by the $k$ th user; $s_{k}(t)$ is the deterministic signature waveform assigned to the $k$ th user in the channel; $T$ is the inverse of the data rate or the symbol time interval; $t_{k}$ is the relative offset of $k$ th user, which depends on channel spread; $s^{2}$ is the noise power spectral density; $n(t)$ is the additive white Gaussian noise (AWGN) with unit power spectral density; $K$ and $2 M+1$ are the number of users and observed symbols in a block, respectively. In the above system model it is assumed that the data symbols are independent, identically distributed (i.i.d) random variables.

In this paper, we focus on a synchronous CDMA system model. However, the method can be extended to an asynchronous CDMA system by extending the observation interval. Note that the detrimental affect of channel distortion or timing asynchronism can also be incorporated into signature waveform mismatch problem which will be discussed in the following section. The simplified one-shot (i.e. one symbol period) synchronous CDMA model is given by: 


$$
y(t)=\stackrel{̊}{\mathrm{a}}_{k=1}^{K} A_{k} b_{k} s_{k}(t)+\operatorname{sn}(t)
$$

The first processing task in the receiver is sampling the continuous-time received data. Here we assume chip rate sampling, which means $C$ equal-spaced samples are taken from successive time intervals of $T$ seconds, where $C$ is the processing gain. Sampled data is then processed a window of a specific size. This is because symbol of desired and interfering transmissions fall entirely to one symbol interval in synchronous communication. After matched filtering and chip rate sampling, the received signal $\mathbf{y}(m)$ for $m$ th symbol can be expressed as a $C^{\prime} 1$ vector:

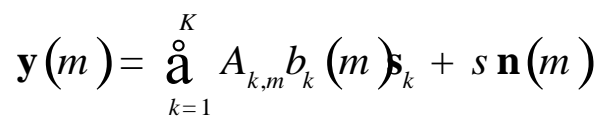

where $\mathbf{s}_{k}$ is the $C^{\prime} 1$ vector representation of $k$ th user's signature sequence and $\mathbf{n}(m)$ is the $C^{\prime} 1$ Gaussian noise vector. Assume that the channel gain of the user signal is not changing relative to bits, therefore $A_{k, m}=A_{k}$. In the remaining sections we will assume that the user 1 is the desired user and the signature code of the desired user is assumed to be known. For example, in the CDMA downlink scenario, the prior signature information of detected user is known in advance.

\section{Blind Detector based on Second-order Cone Programming}

\subsection{The Principle of Blind Detector Based on ICA}

In this subsection, the principle of ICA-based blind detector for CDMA system is illustrated. The representative ICA-based blind detector for CDMA system is shown in Figure 2. The received CDMA signals can be formulated as the mixed observation signals in blind separation model. The effect of channel distortion or timing asynchronization can be formulated as the mixing matrix in blind separation model. Based on this viewpoint, the source signals can be separated or extracted from the received signals using ICAbased blind separation technique. Considering the equation (5), the basic ICA-based BSS model can be acquired as follows,

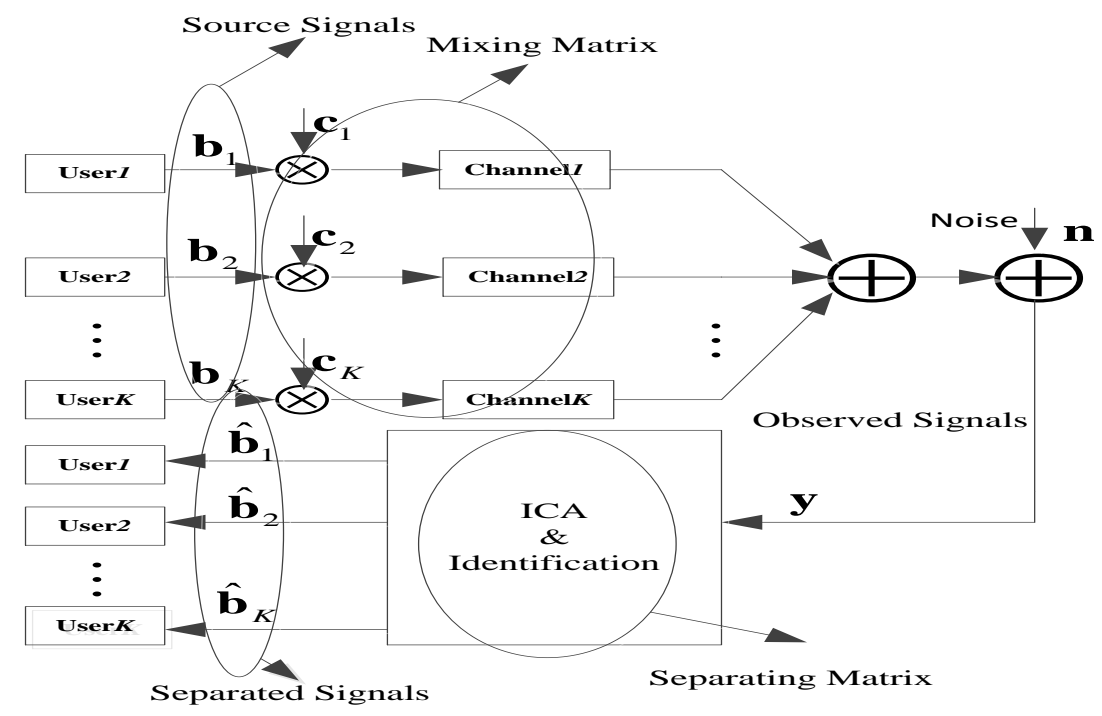

Figure 2. CDMA System Model Based On ICA-Based Blind Detector 


$$
\begin{aligned}
& \mathbf{y}(m)=e_{\mathbf{e}}^{\dot{e}_{1}} A_{1}, \mathbf{s}_{2} A_{2}, \mathrm{~K}, \mathbf{s}_{K} A_{K} \underset{\mathrm{ù}}{\mathrm{u}} \mathbf{b}(m)_{K^{\prime} 1}+s \mathbf{n}(m) \\
& =\mathbf{G b}(m)+s \mathbf{n}(m)
\end{aligned}
$$

In ICA-based CDMA model in $(6), \mathbf{y}(m)$ is the $m$ th observed data vector, $\mathbf{G}$ is an unknown full rank mixing matrix, $\mathbf{b}(m)$ is an unknown non-Gaussian source vector. The goal is to estimate the source $\mathbf{b}(m)$ given only the observations $\mathbf{y}(m)$ and the assumption of the independent sources. That is to say, the estimated source signals $\mathbf{z}=\mathbf{W y}$ are independent and each of them can be used to represent one of the sources. The ICA technique is always based on higher-order statistical (HOS) to formulate cost function. The HOS-based ICA algorithm is actually an unsupervised or neural network learning rule transformed into an efficient fixed-point iteration [1,2]. Afterward, specific features of the received mixed-up signals will be extracted by maximizing their approximate negentropies. Next the principle of the maximizing approximate negentropies will be illustrated so that the separation matrix and source signals can be obtained for blind detection of CDMA systems.

The basic processing of blind separation is to find a certain signal tap and makes $\mathbf{z}_{k}=\mathbf{w}^{H} \mathbf{y}$, where the vector $\mathbf{w}$ is a certain signal tap-weight vector in $\mathbf{W}$ and the superscript $H$ denotes the complex conjugate transposition. This inference is that making the cost function based on the maximum of non-Gaussian is given by as follows $[1,2]$.

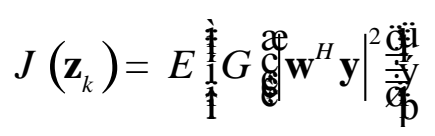

Then, maximizing (10) subject to unit variance constraint $E\left\{\left|\mathbf{w}^{H} \mathbf{y}\right|^{2}\right\}=1$, the weight vector $\mathbf{w}$ of the classical complex-valued FastICA can be obtain the optimization problem.

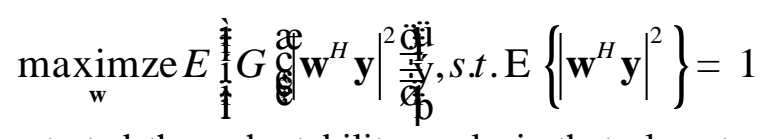

It has been demonstrated through stability analysis that almost any nonquadratic, even function can be regarded as a cost function for non-Gaussianity maximization. For superGaussian signals, a suitable choice of the $G$ function is a smooth, even function with fast computation, such as [1,27]

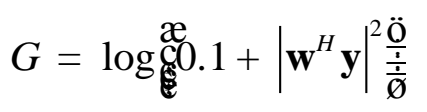

Independent components with super-Gasussian distribution tend to be found first.

To achieve distinct convergent points, a Gram-Schmidt-like orthogonalization technique is generally applied after every iteration step to separate the signals of each user. Available information can be used to solve the problem of weight order ambiguity, either by indirectly choosing appropriate close point initializing ICA iterations or by directly adding the additional constraint [14-20]. The proposed idea which imposes the additional SOC constraint in the classical complex-valued FastICA algorithm and then initializes iterations using the desired spreading code could attain interference suppression and adaptive technique for blind detection over the condition of channel distortion and time asynchronisms in CDMA systems. 


\subsection{Blind Detector Subject to SOC Constraint}

Taking into account some actual factors, the actual received signature waveform can be modeled as $\hat{\mathbf{s}}_{k}=A_{k}\left(\mathbf{s}_{k}+\mathbf{e}_{k}\right)$, where $\mathbf{e}_{k}$ is the mismatch error vector. Note that $A_{k}$ can be estimated by matching the channel output power with $\left\|\mathbf{S}_{k}\right\|^{2}$ [26]. In this way, we obtain the following normalized received signature waveform:

$$
\overline{\mathbf{s}}_{k}=\mathbf{s}_{k}+\mathbf{e}_{k}
$$

Clearly, $\left\|\mathbf{e}_{k}\right\|^{2}$ is a measure of the magnitude of signal waveform mismatch (SWM). The distortion can be due to asynchronism or multipath fading [26]. For example, in the case of timing asynchronism, we can use Taylor approximation to bound $\mathbf{s}(t+t)-\mathbf{s}(t)$, where $t$ is the timing offset. Hence, the signal waveform mismatch error is easily bound by $\|\mathbf{e}\|=\|\overline{\mathbf{s}}-\mathbf{s}\| £ B C t$, where $\mathbf{B}$ is the upper bound for the derivative of the continuous signature waveform $\mathbf{s}(t)$, and $C$ is the spreading factor. In a multipath environment with an $L$-tap channel response $\mathbf{h}$, the actual received signature waveform is $\overline{\mathbf{s}}_{k}=\mathbf{s}_{k} \ddot{A} \mathbf{h}$. Hence, we can obtain the following bound on the mismatch error vector:

$$
\begin{aligned}
\left\|\mathbf{e}_{k}\right\| & =\left\|\overline{\mathbf{s}}_{k}-\mathbf{s}_{k}\right\| £\left\|\mathbf{s}_{k} \ddot{A} \mathbf{h}-\mathbf{s}_{k}\right\| \\
& £\left\|\mathbf{s}_{k} \ddot{A}\left(\mathbf{h}-\mathbf{h}_{\text {idea }}\right)\right\| \\
& £ \sqrt{L}\left\|\mathbf{h}-\mathbf{h}_{\text {idea }}\right\|
\end{aligned}
$$

where $\mathbf{h}_{\text {idea }}$ denotes the ideal channel response. If the channel has a main line of sight component and small multipath components, then $\left\|\mathbf{h}-\mathbf{h}_{\text {idea }}\right\|$ will be small. Without loss of generality, suppose that user 1 is our desired user whose signature waveform is expressed as $\mathbf{s}_{1}$. We assume the distortion error $\mathbf{e}_{1}$ in the desired signal waveform can be bounded by some constant $d>0$, that is, $\left\|\mathbf{e}_{1}\right\| £ d$. The size of $d$ can be estimated, for example (14). The actual received signal waveform $\overline{\mathbf{s}}_{1}$ can be described as a vector in the set

$$
S_{1}(d)=\left\{\overline{\mathbf{s}}_{1} \mid \overline{\mathbf{s}}_{1}=\mathbf{s}_{1}+\mathbf{e}_{1},\left\|\mathbf{e}_{1}\right\| £ d\right\}
$$

Consider the equation (5), (6) and signature waveform mismatch, then yielding

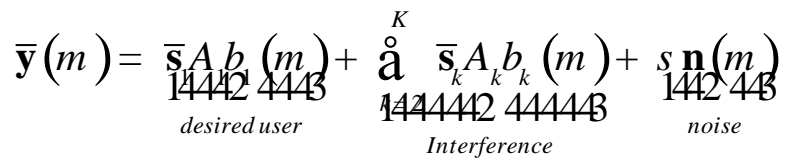

From (16), the first term is the desired user, the second and third terms are the interference vector and noise vector with regard to desired user. For constraint satisfaction, the weight vector $\mathbf{w}$ must satisfy $\left|\mathbf{w}^{H} \overline{\mathbf{S}}_{1}\right|^{3} 1$ for all vectors [26-28]. Such a constraint guarantees that the data symbols of user 1 can be taken from the mixture, regardless of how its signal waveform is mismatched, while the error is bounded by $d$. Now suppose that the weight constraint is enforced; the main goal will be to find a vector $\mathbf{w}$ that maximizes the cost function $\left.E \underset{1}{\stackrel{1}{1}} G \mathbf{w}^{H} \overline{\mathbf{y}}\right|_{\frac{\overline{\mathbf{p}}}{\overrightarrow{3}}} ^{2}$. Then, the problem of worstcase design of the ICA detector based on approximate negentrogy maximization can be 
formulated as

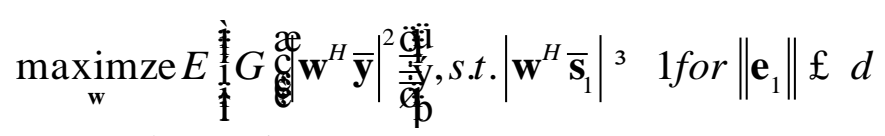

The problem in (17) can be rewritten as

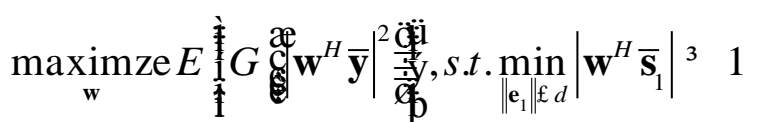

The ICA formulation introduces the additional SOC Programming condition, which has infinitely many nonconvex quadratic constraints on $\mathbf{w}$. According to the derivation of [28, 29], it can be easily shown that in the case of a sufficiently small uncertainty (i.e., where the uncertainty radius $d £\left|\mathbf{w}^{H} \mathbf{s}_{1}\right| /\|\mathbf{w}\|$ ), the left-hand side of the constraint in (18) can be rewritten as

$$
\min _{\left\|\mathbf{e}_{1}\right\| \notin d}\left|\mathbf{w}^{H}\left(\mathbf{s}_{1}+\mathbf{e}_{1}\right)\right|=\left|\mathbf{w}^{H} \mathbf{s}_{1}\right|-d\|\mathbf{w}\|
$$

Using (19) and taking into account that the cost function in (17) is not affected by an arbitrary phase rotation of $\mathbf{w}$, the problem of (18) can be described in this form:

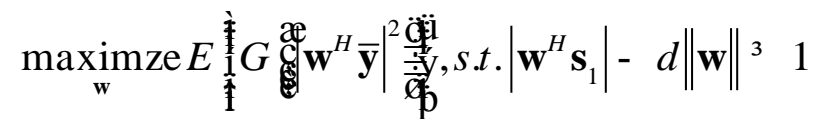

If the cost function in (20) is maximized, the inequality constraint in (20) is satisfied, which means that, while maximizing the cost function in (20), the equality constraint $\left|\mathbf{w}^{H} \mathbf{s}_{1}\right|-d\|\mathbf{w}\|=k$ is satisfied, where $k^{3} 1$. Then, an extra constraint $\operatorname{Im}\left\{\mathbf{w}^{H} \mathbf{s}_{1}\right\}$ can be omitted because the equality constraint $\left|\mathbf{w}^{H} \mathbf{s}_{1}\right|-d\|\mathbf{w}\|=k$ guarantees that the value of $\mathbf{w}^{H} \mathbf{s}_{1}$ is real-valued and positive. Using the equality constraint, the weight vector $\mathbf{W}$ is obtained by rewriting the convex optimization problem of (20) as

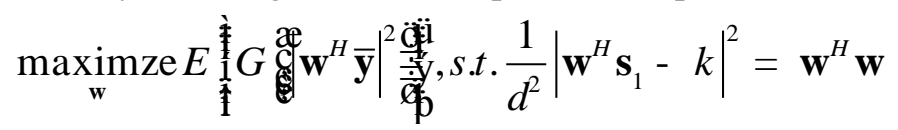

This solves the optimization problem of worst-case performance by maximizing the approximate negentropy subject to the SOC constraint. The constrained optimization problem in (21) will be derived by the quasi-Newton iteration. Newton's method is based on the Lagrangian function

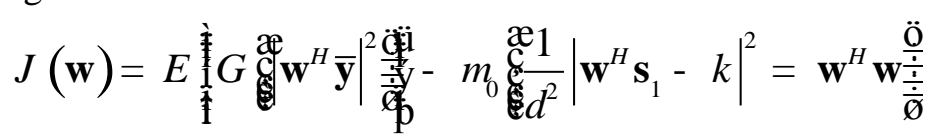

Where $m_{0}$ is a Largrange multiplier. The pre-whitening step is omitted in order to simplify writing.. To use the approximate Newton iteration, the weight-updating algorithm is described as

$$
\begin{aligned}
& \mathbf{w}=\mathbf{w}-\frac{\tilde{\mathrm{N}} J(\mathbf{w})}{\tilde{\mathrm{N}}(\tilde{\mathrm{N}} J(\mathbf{w}(p)))}
\end{aligned}
$$

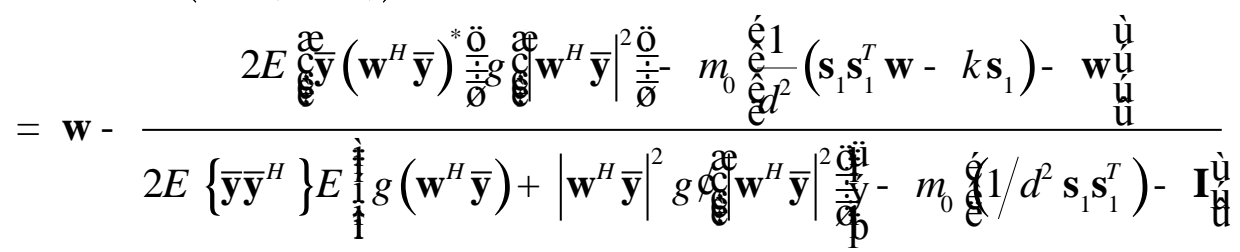

The euqation can be further simplified by multiplying both sides of (23) by 
- $(1 / 2) \mathbf{R}_{\overline{\mathbf{y}}}^{-1} \tilde{\mathrm{N}}(\tilde{\mathrm{N}} J(\mathbf{w}))$. When a sufficiently small $m_{0}$ is chosen, it can be easily shown that the left-hand side of the equality in (23) w multiplying - $(1 / 2) \mathbf{R}_{\overline{\mathbf{y}}}^{-1} \tilde{\mathrm{N}}(\tilde{\mathrm{N}} J(\mathbf{w}))$

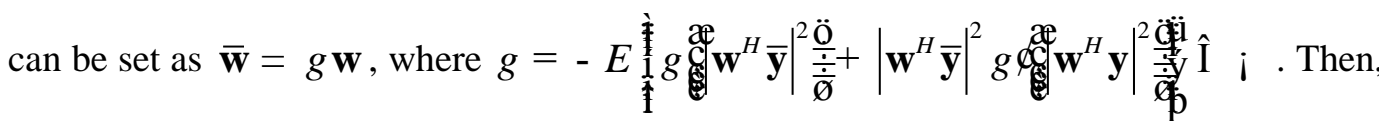
projecting $\overline{\mathbf{w}}$ through $\mathbf{w}(p+1) \neg \overline{\mathbf{w}} / \sqrt{\overline{\mathbf{w}} \mathbf{R}_{\overline{\mathbf{y}}} \overline{\mathbf{w}}}$ could enforce the unit variance constraint $E\left\{\left|\mathbf{w}^{H} \overline{\mathbf{y}}\right|^{2}\right\}=1$ after each ICA iteration. The scalar factor $g$ will not affect the convergence point. Finally, it can be explicitly simplified as

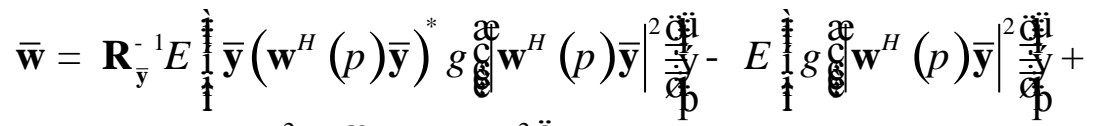

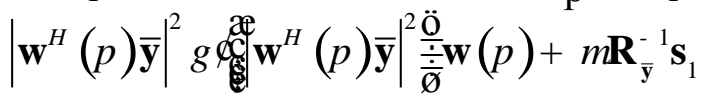

$$
\begin{aligned}
& \mathbf{w}(p+1) \neg \overline{\mathbf{w}} / \sqrt{\overline{\mathbf{w}}^{H} \mathbf{R}_{\overline{\mathbf{y}}} \overline{\mathbf{w}}}
\end{aligned}
$$

Where $m=m_{0} k / 2 d^{2}$. Finally, the desired weight vector with unit variance constraint in the proposed method is as follows.

Step 1. After centering $\overline{\mathbf{y}}$, take a small initial vector $\mathbf{w}(0)=0.01 \mathbf{s}_{1}$. Let iteration number $p=0$.

Step 2. Update

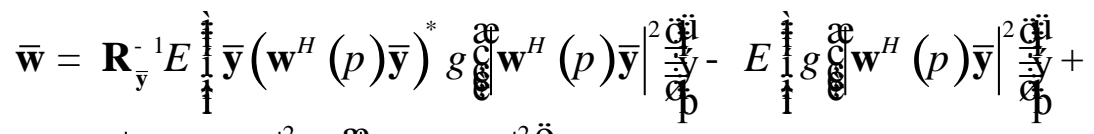

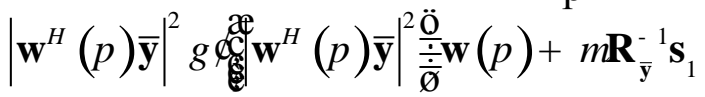

$$
\begin{aligned}
& \mathbf{w}(p+1) \neg \overline{\mathbf{w}} / \sqrt{\overline{\mathbf{w}}^{H} \mathbf{R}_{\overline{\mathbf{y}}} \overline{\mathbf{w}}}
\end{aligned}
$$

Where $\mathbf{R}_{\overline{\mathbf{y}}}=E\left\{\overline{\mathbf{y}} \overline{\mathbf{y}}^{H}\right\}$ is the data correlation matrix and $g$ and $g \notin$ denote the derivatives of $G$ and $g$, respectively.

Step 3. Check the convergence of $\mathbf{w}(p)$. If the error measure is

$\stackrel{\circ}{\mathrm{a}}_{i=1}^{c}\left|w_{i}(p+1)-w_{i}(p)\right|>e$, where $e$ is the terminating error value, let $p=p+1$ and

go back to step 2. Otherwise, output the vector $\mathbf{w}(p)$.

According to classical FastICA, the step that projects $\overline{\mathbf{w}}$ through

$\mathbf{w}(p+1) \neg \overline{\mathbf{w}} / \sqrt{\overline{\mathbf{w}}^{H} \mathbf{R}_{\mathbf{y}} \overline{\mathbf{w}}}$ could enforce the unit variance

constraint $E\left\{\left|\mathbf{w}^{H} \overline{\mathbf{y}}\right|^{2}\right\}=1$ after each step.

In veiw of the blind separation of CDMA system, a modification of the classical FastICA has been proposed that omits the complex pre-whitening step and directly incorporates the SOC constraint into the correct solution. In CDMA system, the proposed SOC-FastICA detection is developed to combat small-to-medium, norm-bounded SWM under colored noise. It is noteworthy that the proposed blind detector can be implemented by choosing a proper value for $m$ without estimating $d$. Due to the algorithmic 
parameter $m$ (including to the Lagrange multiplier $m_{0}$ ), the parameter $k$, and the unknown SWM $d$ ), the proposed SOC-FastICA possesses the capability of self-adjusting computation against SWM. Self-adjusting computation refers to a model of computing where computations can automatically respond to changes in varying $\mathrm{SWM} d$, which means that parameter $m$ can be kept a proper constant under varying circumstances by using self-adjusted $m_{0}$ and $k$ for ICA iterations. The proposed algorithm is nonsensitive to SWM for CDMA systems.

\section{Comparison of Computational Complexity}

The computational complexities of the proposed detector in (24) (including projection) are about $O\left(C^{2} M\right)(M ? C)$ in total, $M$ is the length of data. The computational complexity of the proposed detectors will then be compared with the CMOE detector, the subspace-based MMSE detector, the MMSE-ICA detector, and SOC-JADE. The evaluation of the CMOE detector including autocorrelation matrix $\mathbf{R}_{\overline{\mathbf{y}}}$ and its inverse operation $\mathbf{R}_{\bar{y}}^{-1}$ has the complexity of order. $O\left(C^{2} M\right)+O\left(C^{2}\right)=O\left(C^{2} M\right)$ In the subspace-based MMSE detector, the EVD of autocorrelation matrix has the complexity of $O\left(C^{2} M\right)+O\left(C^{2} K\right)$, and the complexity of the projection of the desired signature waveform onto signal subspace is $O\left(C^{2}\right)$. Thus, the final complexity of the subspacebased MMSE is of order $O\left(C^{2} M\right)$. Besides, in the MMSE-ICA, the computational complexities of autocorrelation matrix, prior subspace estimation, pre-whitening of the received data, and each unit-gain-based ICA iteration are $O\left(C^{2} M\right), O(C K)$, and $O(K M)$, respectively [17]. Thus, the final complexity of the MMSE-ICA is of order $O\left(C^{2} M\right)$. The computational complexities of the SOC-JADE detector are about $O\left(C^{4} M\right)$ in total. The computation burden is mainly dominated by the computation of cumulants [32]. Obviously, the computation complexity of SOC-JADE is higher than the proposed blind detector.

\section{Simulations and Discussions}

To demonstrate the effectiveness of the proposed blind detector, we conduct simulation experiments to evaluate the performance for CDMA systems compared with those of the existing blind detectors, which include the CMOE detector, subspace-MMSE detector, MMSE-ICA detector and SOC-JADE detector. Consider a CDMA system under colored noise environment, using the following parameters. The additive colored noise is modeled by a moving average (MA) process and generated by applying a white Gaussian noise sequence to a corresponding finite impulse response (FIR) filter with transfer function $H(\mathbf{z})=1-a \mathbf{z}^{-1}$, where $a$ is the correlation coefficient. We test the performance of our proposed algorithm on asynchronous or distorted channel CDMA systems. The effect of asynchronism or distorted is described as a SWM problem.

Timing asynchronism or distorted channel is modeled through the presence of SWM as in (13), where $\mathbf{e}_{k}: N\left(0, s^{2}\right)$. Consider CDMA systems using Gold codes of 
length $C=31$ with the number of users $K=7$. For the system with $K=7$ users the interference-to-signal ratio (ISR) is set to be

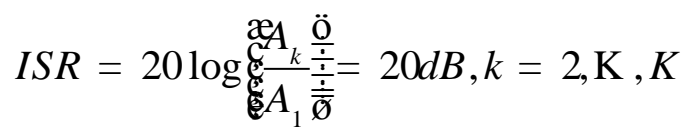

where $A_{k}$ denotes the received signal amplitude (or from the channel gain) of the $k$ th user. This case shows a severe near-far effect. The 1000 symbols are tested in the simulation in order to satisfy the speed of convergence of all the schemes. The results for $K=7$ users are shown in Figure 3, Figure 4 and Figure 5. We test this system with mismatch $d=0.3$. Notice that $d$ is the upper bound on the SWM realization at each random run. We experimented with different $d$ values and results seem to be the same qualitatively. Figure 3 assumes that the value $d$ is known to the detector and we use this value $(\bar{d}=d)$ in the SOC formulation in (20). Figure 4 and Figure 5 show the performance of the proposed method when $\delta$ is overestimated and underestimated, respectively. It can be seen that the proposed detector is robust to errors in estimating the SWM bound. However, the subspace-based MMSE and MMSE-ICA detectors are much more sensitive to the correlated coefficient than the others since parts of the variation of the noise subspace diverge into the signal eigenvectors causing serious destruction to this orthogonality. The blind CMOE cannot capture the desired symbols and diverges after decoding. The SOC-JADE and the proposed blind SOC-FastICA has better performance than other blind detectors. Although the SOC-JADE has a bit of BER performance better than that of the proposed SOC-FastICA due to cumulant properties, the SOC-JADE has higher computation complexity than others. Therefore, the proposed blind detector has superior performance to other blind detectors. The proposed blind detector is promising scheme for real-time blind interference suppression.

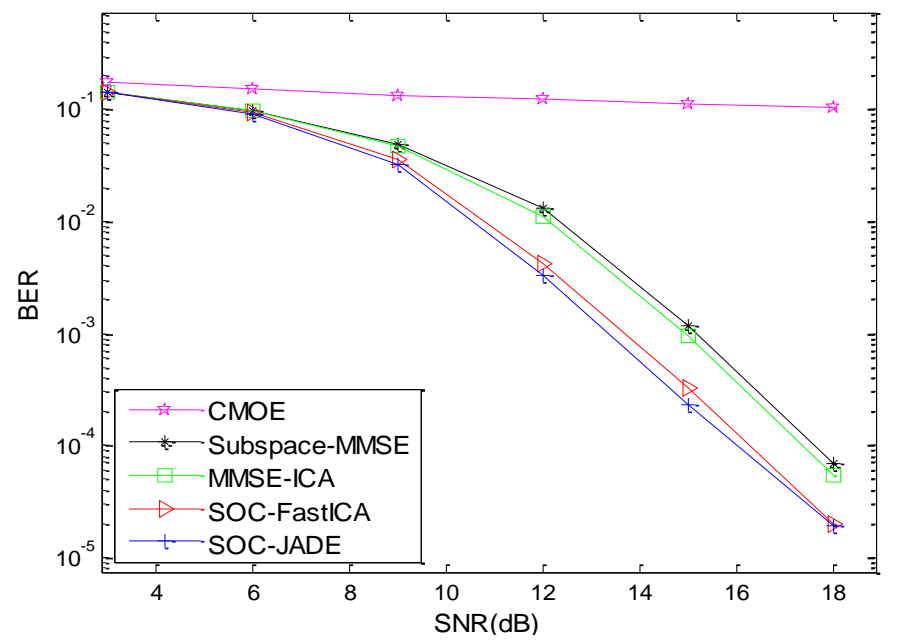

Figure 3. BER versus SNR, Comparison of Different Blind Detectors,

$$
d=0.3 \text { and } \bar{d}=0.3
$$




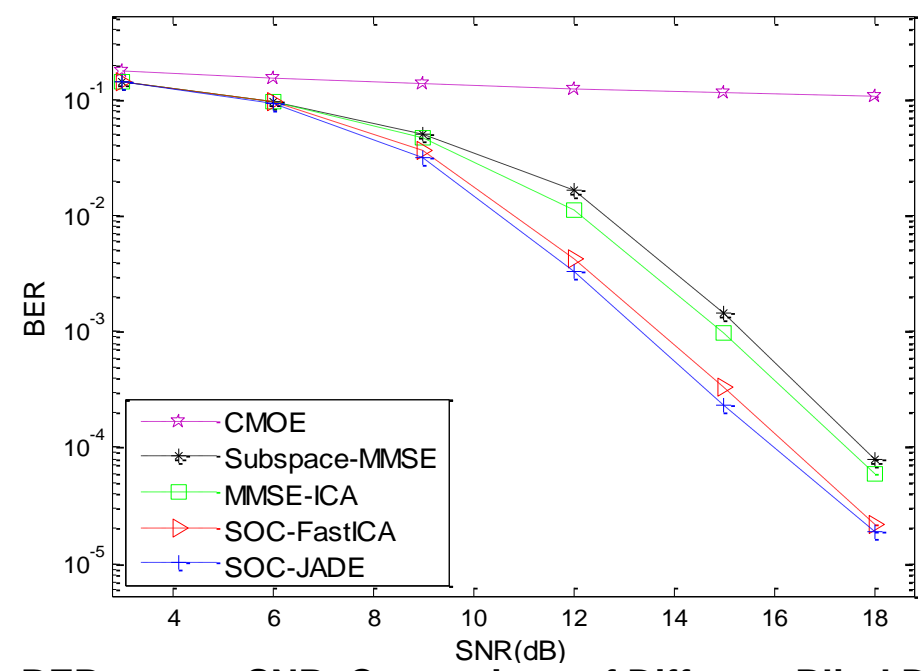

Figure 4. BER versus SNR, Comparison of Different Blind Detectors,

$$
d=0.3 \text { and } \bar{d}=0.5
$$

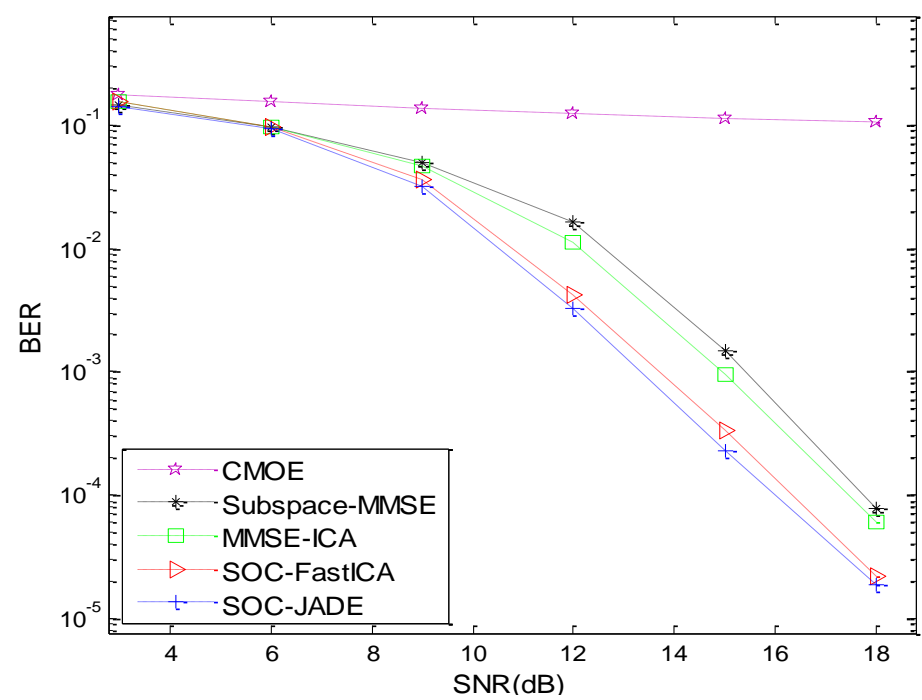

Figure 5. BER versus SNR, Comparison of Different Blind Detectors,

$$
d=0.3 \text { and } \bar{d}=0.1
$$

In order to describe the robustness of the proposed blind detector, the bit error ratio (BER) as a function of SWM mismatch is shown in Figure 6 in SNR $=12 \mathrm{~dB}$. From the Figure 6, we can know that the propose scheme is robust to the SWM problem. In Figure 7, the BER as a function of different symbol numbers is shown for different detector in $\mathrm{SNR}=15 \mathrm{~dB}$. We can know that the proposed blind detector acquires effective performance compared with other detectors. 


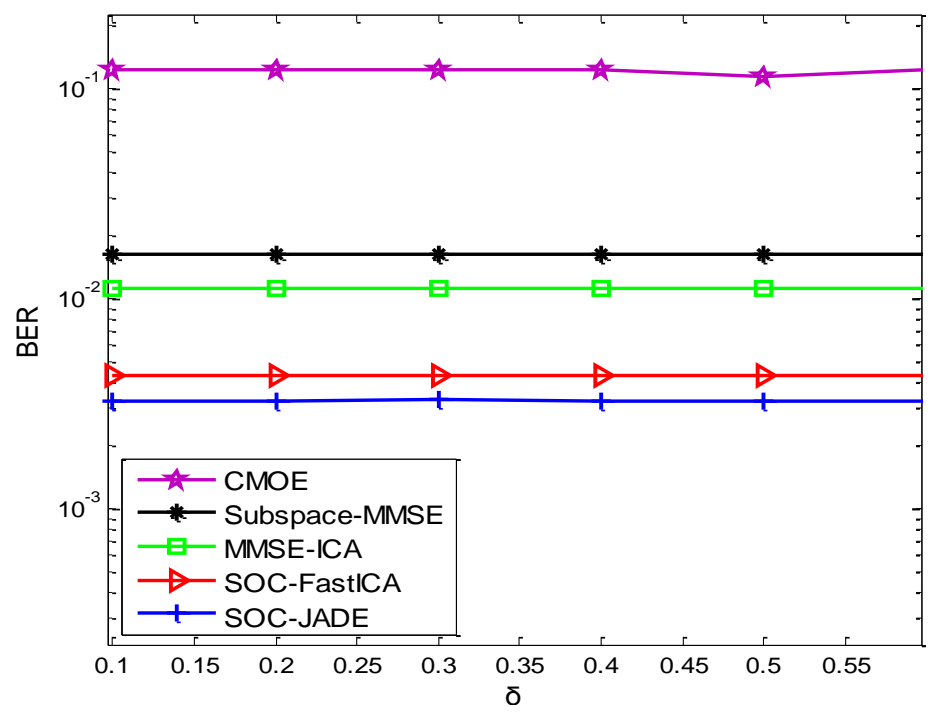

Figure 6. BER versus Different $d$, Comparison of different Blind Detectors

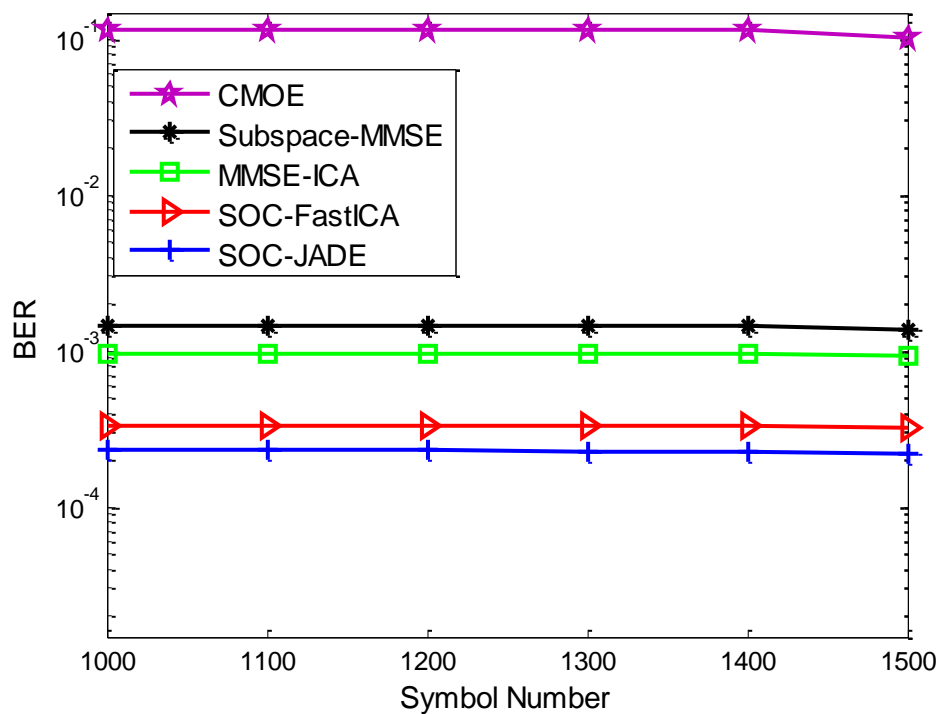

Figure 7. BER versus Different Symbol Number, Comparison of Different Blind Detectors

\section{Conclusions}

As a power technique, ICA can offer additional interference suppression capability, since also independence of the source signals is utilized. ICA can mitigate the performance drops due to erroneous timing and channel estimation problem. As a motivation, in this paper, the ICA-based blind detector for CDMA system is proposed based on approximate negentropy maximization using SOC programming. The proposed blind SOC-FastICA detector can provide robustness against SWM problem and possesses low computation complexity to achieve better performance than the existing blind detectors. Simulation results have demonstrated that the effective performance of the proposed SOC-FastICA detector is obtained. The proposed blind detector is appealing and attractive for the requirements of anti-interference in the future work. 


\section{Acknowledgements}

This work is fully supported by a grant from the national High Technology Research and development Program of China (863 Program) (No. 2012AA01A502), and National Natural Science Foundation of China (No. 61179006), and Science and Technology Support Program of Sichuan Province (No. 2014GZX0004).

\section{References}

[1] A. Hyvärinen, J. Karhunen, and E. Oja, Independent Component Analysis, Wiley, (2001).

[2] E. Bingham, A. Hyvärinen, "A fast fixed-point algorithm for independent component analysis of complex-valued signals", Int. J. of Neural Systems, vol. 10, (2000), pp. 1-8.

[3] J.-F. Cardoso, A. Souloumic, "Blind beamforming for non Gaussian signal", IEE Proceedings- $F$, vol. 140, no. 6, (1993), pp. 362-370,

[4] M. Honig, U. Madhow, S. Verdu, "Blind Adaptive Multiuser Detection", IEEE Trans. Inform. Theory, vol. 41, vo. 7, (1995), pp. 944-966,

[5] L. Hu, X. Zhou, L. Zhang, "Blind multiuser detection based on Tikhonov regularization", IEEE Commun. Lett., vol. 15, no. 5, (2011), pp. 482-483,

[6] Z. Xu, P. Liu, X. Wang, "Blind muliuser detection: from MOE to subspace methods", IEEE Trans. Signal Process., vol. 52, no. 2, (2004), pp. 510-524,

[7] H. Xiaodong Wang, V. Poor, "Blind multiuser detection: a subspace approach", IEEE Trans. Inform. Theory, vol. 44, no. 2, (1998), pp. 677-690,

[8] M. C. Dogan, J. M. Mendel, "Applications of cumulants to array processing. I. Aperture extension and array calibration”, IEEE Trans. Signal Process., vol.43, no. 5, (1995), pp. 1200-1216.

[9] Y. Ma, K.H. Li, A.C. Kot, G. Ye, "A blind code timing estimator and its implementation for DS-CDMA signals in unknown colored noise", IEEE Trans. Veh. Technol., vol. 51, no. 6, (2002), pp. 1600-1607,

[10] A. Belouchrani, M. Amin, "A two-sensor array blind beamformer for direct sequence spread spectrum communications", IEEE Trans. on Signal Processing, vol. 47, (1999), pp. 2191-2199.

[11] A. Belouchrani, M. Amin, "Jammer mitigation in spread spectrum communications using blind source separation”, Signal Processing, vol. 80, (2000), pp. 723-729.

[12] T. Ristaniemi, K. Raju, and J. Karhunen, "Jammer mitigation in DS-CDMA array system using independent component analysis", Proceedings of IEEE Int. Conf. on Communications (ICC), (2002), April; New York, USA.

[13] K. Raju, T. Ristaniemi, J. Karhunen, and E. Oja, "Jammer Suppression in DS-CDMA Arrays Using Independent Component Analysis", IEEE Trans. on Wireless Commun., vol. 5. no. 1, (2006), pp. 1-6.

[14] Jiang Zhang, Hang Zhang, Zhifu Cui, "Dual-antenna-based blind joint hostile jamming cancellation and multi-user detection for uplink of asynchronous direct-sequence code-division multiple access systems", IET Commun., vol. 7, no. 10, (2013), pp. 911-921,

[15] Tapani Ristaniemi, Jyrki Joutsensalo, "Independent Component Analysis with Code Information Utilization in DS-CDMA Signal Separation", Proceedings of IEEE Global Telecommunications Conference (GLOBECOM), (1999), 05 Dec; Rio de Janeireo, pp. 320-324.

[16] Tapani Ristaniemi, Jyrki Joutsensalo, "Advanced ICA-based receivers for DS-CDMA systems", Proceedings of $11^{\text {th }}$ IEEE International Symposium on Personal, Indoor, and Mobile Radio Communications (PIMRC), (2000), London, vol. 1, pp. 276-281.

[17] Tapani Ristaniemi, Jyrki Joutsensalo, "Advanced ICA-based receivers for block fading DS-CDMA channels", signal processing, no. 82, (2002), pp. 471-431.

[18] Malay Gupta, Balu Santhanam, "ICA Based Blind Adaptive MAI Suppression in DS-CDMA Systems", Proceedings of $11^{\text {th }}$ IEEE Digital Signal Processing\& Signal Processing Education Workshop, (2004), 1-4 Aug., pp. 201-205.

[19] W.Y. Leong, J. Homer, "Blind Multiuser Receiver for DS-CDMA Wireless System", IEE Proc. Commun., vol. 153, no. 5, (2006), pp. 733-739.

[20] Fengbo Lu, Zhitao Huang, Wenli Jiang, "Blind estimation of spreading sequence of CDMA signals based on FastICA and performance analysis", Journal on Communications, vol. 32, no. 8, (2011), pp. 136-142.

[21] Xiaotian Ren, Hui Xu, Zhitao Huang, Fengbo Lu, "FastICA Based Blind Estimation of Spreading and Information Seuqences of Short-code CDMA Signals in Synchronous and asynchronous Systems", ACTA ELECTRONICA SINICA, vol. 39, no. 12, (2011), pp. 2726-2732.

[22] Xiaotian Ren, Zhitao Huang, Fenghua Wang, Fengbo Lu, "FastICA based Optimize Blind Estimation of Spreading Sequence of CDMA Signals", ACTA ELECTRONICA SINICA, vol. 40, no. 8, (2012), pp. 1532-1538.

[23] M. G. S. Sriyananda, J. Joutsensalo, and T. Hämäläinen, "Interference Cancellation Scheme for Spread Spectrum Systems with Blind Principles", Proceedings of $27^{\text {th }}$ IEEE International Conference on Advanced Information Networking and Applications, (2013), 25-28 March, Barcelona, pp. 1078-1082.

[24] Zhongqiang Luo, Lidong Zhu, Chengjie Li, "Exploiting Charrelation Matrix to Improve Blind 
Separation Performance in DS-CDMA Systems", Proceedings of the $9^{\text {th }}$ International Conference on Communications and Networking in China (CHINACOM), (2014), 14-16 Aug.; Maoming, China, pp. 369- 372.

[25] Mohammad Eslami, Javad Afshar Jahanshahi, and Seyed Ali Ghorashi, "Joint ICA-Based Blind Detection and Parameter Assessment in DS-CDMA Systems", Journal of Applied Sciences, Engineering and Technology, vol. 3, no. 9, (2011), pp. 857-865.

[26] Shuguang Cui, Mikalai Kisialion, Zhi-Quan Luo, and Zhi Ding, "Robust Blind Multiuser Detection Against Signature Waveform Mismatch Based on Second-Order Cone Programming", IEEE Trans. on Wireless Commun., vol. 4, no. 4, (2005), pp. 1285-1291.

[27] Chih-Wei jen, Shyh-Jye Jou, "Blind ICA detection based on second-order cone programming for MCCDMA systems", Eurasip Journal on Advances in Signal Processing, vol. 151, (2014), pp. 1-14.

[28] Z.-Q. Luo, W. Yu, "An introduction to convex optimization for communications and signal processing", IEEE J. Select Area Commun., vol. 24, no. 8, (2006), pp. 1426-1438.

[29] D. Blanco, B. Mulgrew, "ICA in signals with multiplicative noise", IEEE Trans. Signal Process., vol. 52, no. 8, (2005), pp. 2648-2657.

[30] W. Y. Leong, D. P. Mandic, "Noisy component extraction", IEEE Trans. Circuit Syst, vol. 57, no. 3, (2010), pp. 664-671.

[31] Jeffrey G. Andrews, Stefano Buzzi, Wan Choi, etc, "What Will 5G be?", IEEE JSAC Special Issue on 5G Wireless Communication Systems, (2014), pp. 1-17.

\begin{abstract}
Authors
Zhongqiang Luo, he received the B.S. and M.S. degrees in communication engineering from Sichuan University of Science \& Engineering, Zigong, China, in 2009 and 2012, respectively. Now he is studying for Ph.D. degress in National Key Lab. of Science and Technology on Communications of University of Electronic Science and Technology of China, Chengdu, China. His research interests include blind source separation, wireless communication system. *The corresponding author Email: luozhongqiang@126.com

Chengjie Li, he received the B.Sc. degree in Shandong normal University, Qufu (Confucius's hometown), China, 2004, the M.Sc. degree in computer software and theory in Xihua University, Chengdu, China, 2009. Now Chengjie Li is studying for Ph.D. degree in Communication and Information System in University of Electronic Science and Technology of China (UESTC), Chengdu, China, 2013. His research interests include blind source separation, data mining and intelligent information processing.
\end{abstract}


International Journal of Future Generation Communication and Networking Vol. 9, No.9, (2016) 